\title{
Correction to: Effects of active molecules of Korean pine seed on rodent health and implications for forest regeneration
}

\author{
Gang Wei ${ }^{1} \cdot K_{\text {Ke Rong }}^{2} \cdot$ Kexin Yang ${ }^{1}$ Zhiying Bao ${ }^{1}$. \\ Xiaotong Zhang ${ }^{1} \cdot$ Zhi Zhang $^{1} \cdot$ Yanni Gong ${ }^{3}$. \\ Jiafu Wang ${ }^{4}$
}

Published online: 31 October 2021

(C) Northeast Forestry University 2021

\section{Correction to: J. For. Res. \\ https://doi.org/10.1007/s11676-021- \\ 01380-2}

In the Original publication, the author has found that Table 8 has been inadvertently published with errors due to incorrect calculation. The corrected Table 8 is provided below:

\section{Effect of PNO supplements on serum lipid levels} and the atherosclerosis index

TC, TG and LDL-C levels increased, while the HDL-C levels decreased notably in the HFD group compared to the ND group $(P<0.01$; Table 8$)$. After supplementation with PNO, serum lipid (TG, TC) levels of mice in the $\mathrm{M}-\mathrm{PNO}$ and H-PNO groups decreased; the differences were

statistically significant compared with those in the HFD group $(P<0.05)$. Furthermore, PNO supplements significantly improved HDL-C levels and decreased LDL-C levels $(P<0.01)$.

The HFD group had significantly higher atherosclerotic index $\left(\mathrm{A}_{\mathrm{AI}}\right)$ values than the ND group. Feeding with a highfat diet (HFD) could significantly increase the incidence of atherosclerotic disease in mice. The PNO group showed a decrease in the $\mathrm{A}_{\mathrm{AI}}$ index of the HFD group by $68 \%, 82 \%$ and $67 \%$, respectively $(P<0.01)$. $\mathrm{A}_{\mathrm{AI}}$ indicates the degree of atherosclerosis and occurrence of CVD.

The original article can be found online at https://doi.org/10. 1007/s11676-021-01380-2.

Zhi Zhang

ldzhangzhi@126.com

Yanni Gong

369909311@qq.com

1 School of Forestry, Northeast Forestry University, Harbin 150040, People's Republic of China

2 College of Wildlife and Protected Area, Northeast Forestry University, Harbin 150040, People's Republic of China

3 Beidahuang Wondersun Dairy Co. Ltd, Harbin 150040, People's Republic of China

4 Inner Mongolia Greater, Khingan Range Forestry Science and Technology Research Institute, Yakeshi 022150,

People's Republic of China 
Table 8 Effect of PNO on lipid levels and the atherosclerosis index in hyperlipidemic mice

\begin{tabular}{llllll}
\hline Group & \multicolumn{2}{l}{ Concentration $(\mathrm{mmol} / \mathrm{L})$} & \multirow{2}{*}{$\mathrm{A}_{\mathrm{AI}}$} \\
\cline { 2 - 5 } & $\mathrm{TC}$ & $\mathrm{TG}$ & HDL-C & LDL-C & \\
\hline ND & $4.04 \pm 0.33$ & $1.44 \pm 0.35$ & $3.17 \pm 0.19$ & $1.37 \pm 0.31$ & $0.27 \pm 0.14$ \\
HFD & $6.64 \pm 0.67^{\& \&}$ & $2.88 \pm 0.34^{\&}$ & $1.24 \pm 0.15^{\& \&}$ & $2.98 \pm 0.12^{\& \&}$ & $4.35 \pm 0.09^{\& \&}$ \\
SG & $5.17 \pm 0.34^{\& \& * *}$ & $2.21 \pm 0.34$ & $2.84 \pm 0.11^{\& *}$ & $1.88 \pm 0.68^{* *}$ & $0.82 \pm 0.15^{\& \& * *}$ \\
L-PNO & $5.59 \pm 0.54^{\& \& * *}$ & $1.52 \pm 0.27^{*}$ & $2.34 \pm 0.51^{\& \& * *}$ & $2.06 \pm 0.19^{* *}$ & $1.39 \pm 0.11^{\& \& * *}$ \\
M-PNO & $4.27 \pm 0.11^{* *}$ & $1.17 \pm 0.73^{*}$ & $2.39 \pm 0.15^{\& \& * *}$ & $1.76 \pm 0.05^{* *}$ & $0.79 \pm 0.21^{\& \& * *}$ \\
H-PNO & $5.61 \pm 0.24^{\& \& *}$ & $1.28 \pm 0.30^{*}$ & $2.32 \pm 0.28^{\& \& * *}$ & $1.82 \pm 0.18^{* *}$ & $1.42 \pm 0.13^{\& \& * *}$ \\
\hline
\end{tabular}

Data are expressed as mean $\pm \mathrm{SD}(\mathrm{n}=10)$

${ }^{\&}$ Represents $P<0.05$ vs ND

*Represents $P<0.05$ vs HFD

Publisher's Note Springer Nature remains neutral with regard to jurisdictional claims in published maps and institutional affiliations. 